\title{
IL-12 suppression, enhanced endocytosis and up-regulation of MHC-II and CD80 in dendritic cells during experimental endotoxin tolerance
}

Jing ZHANG ${ }^{1}$, Jie-ming $\mathrm{QU}^{2, *}$, Li-xian $\mathrm{HE}^{1}$

${ }^{1}$ Department of Pulmonary Medicine, Zhongshan Hospital, Fudan University, Shanghai 200032, China; ${ }^{2}$ Department of Pulmonary Medicine, Huadong Hospital, Fudan University, Shanghai 200040, China

Aim: The aim of this study was to investigate endocytosis, MHC-II expression and co-stimulatory molecule expression, as well as interleukin-12 (IL-12) production, in bone marrow dendritic cells (DCs) derived from endotoxin tolerant mice.

Methods: Endotoxin tolerance was induced in C57BL/10J mice through four consecutive daily intraperitoneal injections of $1.0 \mathrm{mg} / \mathrm{kg}$ of 055:B5 Escherichia coli lipopolysaccharide (LPS). Bone marrow DCs were isolated in the presence of GM-CSF and IL-4 and purified by anti-CD11c Micro beads. FITC-dextran uptake by DCs was tested by flow cytometric analysis and the percentage of dextran-containing cells was calculated using a fluorescence microscope. The expression of surface MHC-II, CD40, CD80, and CD86 was also detected by flow cytometric analysis. An ELISA was used for the measurement of IL-12 production by DCs with or without LPS stimulation.

Results: Endotoxin tolerance was successfully induced in C57BL/10J mice, evidenced by an attenuated elevation of systemic TNF-a. DCs from endotoxin tolerant mice possessed enhanced dextran endocytosis ability. The expression of surface MHC-II and CD80 was higher in DCs from endotoxin tolerant mice than in DCs from control mice, whereas the expression of CD40 and CD86 was not altered. Compared with DCs from normal control mice, DCs from endotoxin tolerant mice produced less IL-12 after subsequent in vitro stimulation with LPS.

Conclusion: These data suggest enhanced endocytosis, selective up-regulation of MHC-II and CD80 and IL-12 suppression in DCs during in vivo induction of endotoxin tolerance.

Keywords: endotoxin tolerance; dendritic cells; endocytosis; MHC-II; co-stimulatory molecules; interleukin-12

Acta Pharmacologica Sinica (2009) 30: 582-588; doi: 10.1038/aps.2009.34; published online 6th April 2009

\section{Introduction}

Lipopolysaccharide (LPS)-containing endotoxins are potent activators of the innate immune system and are central to the pathological excessive or deregulated inflammation observed in Gram-negative sepsis. The endotoxins can lead to systemic inflammatory response syndrome, multiple organ failure and even death ${ }^{[1,2]}$. Therefore, the prevention of excessive release of pro-inflammatory mediators through medications such as corticosteroid and anti-tumor necrosis factor- $\alpha$ (TNF- $\alpha$ ) antibody is one of the vital strategies for the prevention and treatment of tissue injury in this clini-

* Correspondence to Prof Jie-ming QU.

E-mail jmqu64@yahoo.com.cn

Received 2008-11-16 Accepted 2009-03-04 cal setting ${ }^{[1-3]}$. However, none of these treatments showed significant improvement in clinical trials ${ }^{[4]}$. In part, the benefit of the suppression of proinflammatory mediators is thought to be counteracted by the all-around inhibition of the immune system.

Consecutive low doses of LPS can induce endotoxin tolerance in which endotoxin triggered responses are at least partially abrogated ${ }^{[5]}$. In vitro, the endotoxin-tolerant monocytes and macrophages are characterized by inhibition of LPS-stimulated proinflammatory cytokine production ${ }^{[5-7]}$. Our previous study demonstrated that endotoxin-tolerant animals had a marked reduction in the systemic response to endotoxemia, including mortality, weight loss, fever and serum cytokine level ${ }^{[8]}$, in accordance with other in vivo studies $^{[5,7,9-11]}$. Moreover, several previous studies showed that the repetitive low-dose LPS pretreated mice had 
improved survival after bacterial and fungal infection ${ }^{[12,13]}$. The fundamental mechanisms that underlie such beneficial effects of endotoxin tolerance have been increasingly studied recently and might be associated with the reprogramming of immune cells during LPS pretreatment.

Dendritic cells (DCs) are composed of different subgroups and coordinate the innate and the adaptive arms of the immune response. They are scattered throughout the body, where they work as "immunological sensors" that are capable of decoding the dangerous signals, presenting the information to $\mathrm{T}$ cells and generating the right class of immune responses ${ }^{[14-18]}$. Currently, little is known about the variation of DCs during the induction of endotoxin tolerance. To develop a greater understanding of the alteration of DCs during LPS pretreatment, we reproduced murine endotoxin tolerance through repetitive intraperitoneal injections of low dose LPS and investigated three functions of isolated bone marrow DCs: (1) endocytotic capacity; (2) expression of MHC-II and co-stimulatory molecules on the cell surface; and (3) the ability to produce interleukin-12 (IL-12).

\section{Materials and methods}

Animals and treatment Specific pathogen-free male C57BL/10J mice at 4-6 weeks of age were purchased from Shanghai SLAC Laboratory Animal Inc/National Rodent Laboratory Animal Resources, Shanghai branch, and bred under specific pathogen-free conditions in the animal facility of the Liver Cancer Institute of Zhongshan Hospital, Fudan University. The procedures involving animals and their care were conducted in conformity with national and international laws and policies.

The mice were randomly divided into the endotoxin tolerant group, which was pretreated with an intraperitoneal injection of $1 \mathrm{mg} / \mathrm{kg}$ Escherichia coli LPS (serotype 055:B5; Sigma, St Louis, MO) at the same time on each of four consecutive days, and the normal control group, which was pretreated with intraperitoneal injections with the same volume of pyrogen-free $0.9 \%$ sodium chloride. To evaluate the systematic inflammatory response to subsequent LPS challenge, a dose of $10 \mathrm{mg} / \mathrm{kg}$ LPS was injected intraperitoneally on the fifth day. Each group was divided into two subgroups ( 6 animals of each subgroup). One subgroup received a high dose LPS challenge and samples were obtained $6 \mathrm{~h}$ after high dose LPS injection, and the other subgroup received sterile normal saline, serving as a $0 \mathrm{~h}$ control. The mice were sacrificed and samples of blood were drawn from the inferior vena cava. Blood was centrifuged at $2000 \mathrm{r} / \mathrm{min}$ for $10 \mathrm{~min}$ and serum was stored at $-80^{\circ} \mathrm{C}$ for the TNF- $\alpha$ assay.
TNF- $\boldsymbol{\alpha}$ determination TNF- $\alpha$ was measured with a commercially available sandwich ELISA kit (Senxiong Company, Shanghai, China) according to the manufacturer's guidelines. The lowest detection limit was $62.5 \mathrm{pg} / \mathrm{mL}$.

Isolation and cultures of DCs After four daily injections of LPS or $0.9 \%$ sodium chloride, mice were sacrificed on the fifth day. DCs were generated from bone marrow precursors harvested in RPMI-1640 (Hyclone-Pierce) medium containing $10 \%$ fetal bovine serum (FBS), $100 \mathrm{U} /$ $\mathrm{mL}$ penicillin, $100 \mu \mathrm{g} / \mathrm{mL}$ streptomycin, $10 \mathrm{ng} / \mathrm{mL}$ recombinant murine interleukin- 4 and $20 \mathrm{ng} / \mathrm{mL}$ recombinant murine granulocyte-macrophage colony-stimulating factor (all from Sigma, St Louis, Mo) as previously described ${ }^{[19]}$. DCs were enriched by adding $100 \mu \mathrm{L}$ of anti-CD11c Micro beads (Milteny Biotec) per $10^{8}$ cells for $15 \mathrm{~min}$ at $4{ }^{\circ} \mathrm{C}$, followed by washing and positive selection using autoMACS (Milteny Biotec), according to the manufacturer's protocol. This resulted in $95 \%-98 \%$ pure $\mathrm{CD} 11 \mathrm{c}^{+}$cells as determined by flow cytometry. The morphology of the collected cells was observed under a light microscope after Giemsa stain and under a scanning electron microscope as a supplementary confirmation of DCs. Cell viability was over $95 \%$ as examined using trypan blue. For each group, three batches of bone marrow DCs were harvested from different individual animals for subsequent investigations.

Endocytosis assay Freshly isolated DCs $\left(2 \times 10^{5}\right)$ from control and tolerant mice were incubated with $1 \mathrm{mg}$ fluorescein isothiocyanate (FITC)-conjugated-dextran $(4 \mathrm{kDa}$, Sigma, St Louis, MO) in a total volume of $1 \mathrm{~mL}$ for three hours at $37^{\circ} \mathrm{C}$. As a negative control, cells were incubated under the same conditions at $4{ }^{\circ} \mathrm{C}$. Uptake was then stopped by the addition of ice-cold PBA (PBS containing 1\% bovine serum albumin and $0.1 \%$ sodium azide). The level of endocytosis was evaluated by two different methods. In the first, cells were washed twice with PBA and then analyzed with FACScan flow cytometry. In the second, cells were washed, cytocentrifuged onto glass slides, and quantified by fluorescence microscopy. Ten random fields were assessed per slide, and the percentage of dextran-containing cells was determined. Results are expressed as the median (25th percentile, 75 th percentile) of the percentage ingestion.

Flow cytometric analysis of DCs Flow cytometry was performed to analyze DCs for expression of cellular surface molecules. Briefly, DCs were washed with PBA and then $0.05 \mathrm{~mL}$ medium containing $1 \times 10^{5}$ cells was incubated with $10 \mathrm{ng} / \mathrm{mL}$ of FITC-conjugated monoclonal antibodies (mAb) to MHC-II, CD40, CD80, and CD86, respectively, for $30 \mathrm{~min}$ at $4{ }^{\circ} \mathrm{C}$. This was followed by extensive washing and analysis on cells gated to exclude nonviable cells. The 
expression of the surface proteins was analyzed as the percentage of positive cells in the relevant population. An isotype-matched FITC-conjugated $\mathrm{mAb}$ for samples labeled in parallel was used as a control (all antibodies from Pharmingen, San Diego, CA, USA). All experiments were performed at least in triplicate.

IL-12 production of DCs Purified DCs were diluted to $2 \times 10^{6}$ cells $/ \mathrm{mL}$ and incubated in the presence or absence of LPS $(1 \mu \mathrm{g} / \mathrm{mL})$ for $24 \mathrm{~h}$. The supernatants were collected and cleared by centrifugation and then stored at $-80^{\circ} \mathrm{C}$. The concentration of IL-12 was measured with the commercially available sandwich ELISA kit (Senxiong Company, Shanghai, China) according to the manufacturer's guidelines. The lowest detection limit was $62.5 \mathrm{pg} / \mathrm{mL}$.

Statistics Statistics were performed by Prism 5 for Windows. Results were expressed as medians (25th percentile, 75th percentile). A Mann-Whitney U-test or Kruskal-Wallis one-way analysis of variance by ranks was adopted to calculate statistical differences between two groups or among 3 or more groups, respectively. Differences were considered significant at the level of $P<0.05$.

\section{Results}

The reproduction of endotoxin tolerance After the intraperitoneal injection of $10 \mathrm{mg} / \mathrm{kg}$ LPS, normal control mice showed less activity and food intake and faster respiratory rate. Such abnormality was not found in the endotoxin tolerant mice.

As shown in Figure 1, the normal control group responded to a high dose of LPS with a significant increase in serum TNF- $\alpha$ from $126.0(97.91,128.5) \mathrm{pg} / \mathrm{mL}$ at $0 \mathrm{~h}$ to $296.2(249.3,323.0) \mathrm{pg} / \mathrm{mL}$ at $6 \mathrm{~h}(P<0.05)$. However, the serum TNF- $\alpha$ was significantly blunted in the endotoxin tolerant group, implying systemic endotoxin tolerance.

Enhanced endocytosis capacity of DCs from endotoxin tolerant mice To examine whether the ability of DCs to capture antigens was affected by endotoxin tolerance, we tested the endocytotic activity of bone marrow DCs by measuring the uptake of FITC-conjugated dextran particles by flow cytometry and fluorescence microscope observation. In accordance with more FITC positive cells as determined by a FACScan flow cytometry (Figure 2A), the enriched DCs obtained from endotoxin tolerant mice had an elevated percentage ingestion $[59.6(53.6,64.2) \%]$ as compared with the control mice $[34.5(31.0,39.8) \%, P<0.05]$ under a fluorescence microscope (Figure 2B-2D). These results indicated an enhanced endocytosis capacity against dextran in DCs

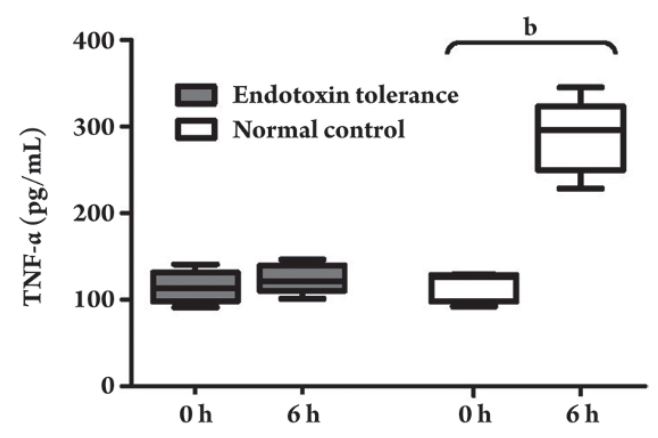

Figure 1. The blocked elevation of serum TNF- $\alpha$ in the endotoxin tolerant group after a high dose LPS challenge. To determine whether the four consecutive daily injections of $1 \mathrm{mg} / \mathrm{kg}$ LPS induced endotoxin tolerance successfully, we investigated the variation of serum TNF- $\alpha$ production between 0 and $6 \mathrm{~h}$ after $10 \mathrm{mg} / \mathrm{kg}$ LPS injection on the fifth day in the endotoxin tolerant group and in the normal control group. A sandwich ELISA was used to measure the concentration of TNF- $\alpha$. The results are expressed in picograms per milliliter. Data were obtained from six mice for each time point in each group. ${ }^{b} P<0.05$.

from endotoxin tolerant mice.

Altered expression of MHC-II and T cell co-stimulatory molecules on DCs from endotoxin tolerant mice Optimal antigen presentation requires the expression of MHC-II and co-stimulatory molecules on DCs ${ }^{[17,18]}$. Thus, we tested the expression of MHC-II, CD40, CD80, and CD86 on the cell surface using flow cytometric analysis. As shown in Figure 3, DCs constitutively expressed these molecules; moreover, endotoxin tolerance induces the upregulation of MHC-II and CD80 $(P<0.05)$. However, the expression of CD40 and CD86 was almost unaltered. These results indicated that MHC-II and CD80 were selectively upregulated during low-dose LPS pretreatment.

Impaired ability of DCs from low-dose LPS pretreated mice to produce IL-12 in response to subsequent LPS exposure in vitro We investigated the ability of purified DCs to produce IL-12 by quantitatively measuring the protein concentration in the supernatants of the cell culture. As shown in Figure 4, DCs from mice pretreated in vivo with repetitive low-dose LPS produced less IL-12 after subsequent in vitro stimulation with LPS $[121.7$ (106.2, 138.9) $\mathrm{pg} / \mathrm{mL}$ ], compared with DCs from control mice [346.1 (308.6, 363.7) pg/mL, $P<0.05]$. Moreover, DCs from LPSprimed mice also had a tendency to produce less IL-12 than DCs from control mice; after LPS stimulus in vitro, the IL-12 production of DCs in the normal control group was elevated, whereas it was down-regulated in the endotoxin tolerant group. These data suggested a potent suppression of IL-12 production in DCs from endotoxin tolerant mice. 

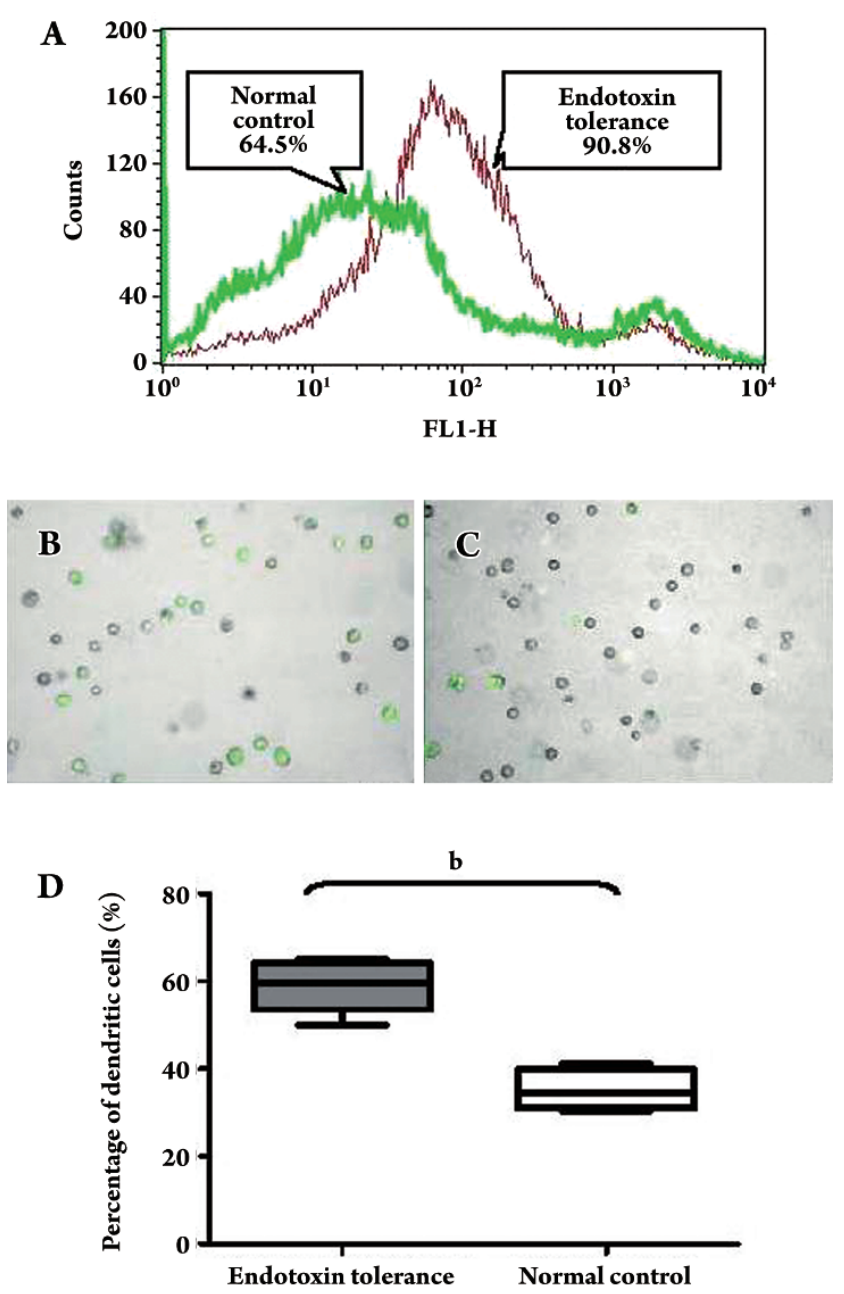

Figure 2. Dendritic cells (DCs) from endotoxin tolerant mice exhibit an increased ability for endocytosis. The in vitro ability of DCs to endocytose dextran was tested by incubating DCs with FITClabeled dextran at a ratio of $2 \times 10^{5}$ cells to $1 \mathrm{mg}$ dextran for three hours. The cells were thereafter examined for endocytotic ability in two ways: (1) flow cytometry (shown in A) and (2) fluorescence microscope quantification after cytocentrifugation onto glass slides. Ten random fields were assessed per slide, and the results are presented as percentage ingestion, ie, the percentage of FITC-dextran containing cells (shown in B-D). All experiments were performed at least in triplicate. (A) Representative example of a histogram of FITC-dextran treated DCs from endotoxin tolerant mice (red line) and normal control mice (green line). (B) One representative photo taken in a fluorescence microscope of DCs from endotoxin tolerant mice. The percentage of DCs containing FITC-dextran was 59\%. (C) One representative photograph of DCs from normal control mice. The percentage ingestion monitored using microscopy was 34\%. (D) Statistical analysis of the data obtained from at least three separate experiments. ${ }^{\mathrm{b}} \mathrm{P}<0.05$. (B, C) $\times 100$.

\section{Discussion}

In this experiment, we induced endotoxin tolerance in
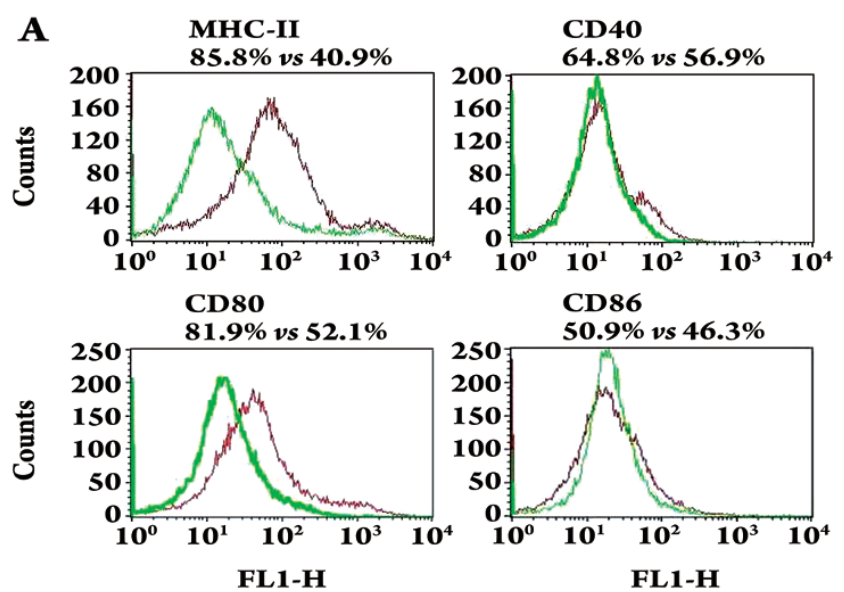

$\mathbf{B}$

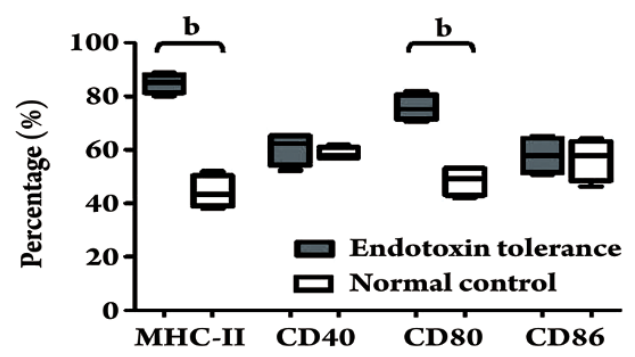

Figure 3. Expression of MHC-II and co-stimulatory molecules on dendritic cells (DCs). Bone marrow DCs were obtained from normal control mice and endotoxin tolerant mice and then stained with $\mathrm{mAbs}$ for FACS analysis. (A) The histograms of a flow cytometric analysis of the expression of the surface molecules MHC-II, CD40, CD80, and CD86 on DCs. The red line and green line marked the positive cells of endotoxin tolerant cells and control cells, respectively, and the percentage of positive cells was indicated. All experiments were performed at least in triplicate, and one representative plot was shown. (B) Statistical analysis of the expression of cellular surface molecules. The results were obtained from at least three separate experiments. ${ }^{\mathrm{b}} \mathrm{P}<0.05$.

C57BL/10J mice with four consecutive daily intraperitoneal injections of $1 \mathrm{mg} / \mathrm{kg}$ LPS for further studies on the alterations of DCs. The successful induction of endotoxin tolerance was confirmed by the blocked systematic TNF- $\alpha$ elevation in response to $10 \mathrm{mg} / \mathrm{kg}$ LPS. TNF- $\alpha$ is one of the central mediators of LPS toxicity, and our previous study ${ }^{[8]}$, together with other experiments ${ }^{[5,20]}$, showed that a block in TNF- $\alpha$ production was a reliable marker of the establishment of endotoxin tolerance.

We adopted the regimen of repetitive low-dose LPS pretreatment to induce endotoxin tolerance rather than a single high-dose LPS challenge because the latter was similar to the hypoinflammatory state, termed "immunological paralysis," in patients with septic shock who have an increased risk of succumbing to a secondary infection ${ }^{[21-23]}$. Furthermore, 


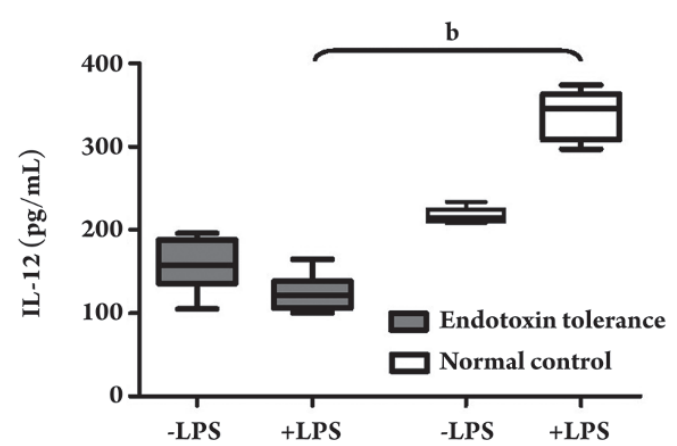

Figure 4. Analysis of IL-12 production by dendritic cells (DCs). The supernatants with or without LPS stimulation were assayed by sandwich ELISA. The IL-12 level was expressed in picograms per milliliter. Data were from at least three separate experiments performed in duplicate for each group. ${ }^{b} P<0.05 v s$ normal control.

endotoxin tolerance induced by low-dose LPS pretreatment could alleviate the severity of infections induced by Pseudomonas aeruginosa ${ }^{[24]}$ and serovar Typhimurium ${ }^{[25]}$ despite attenuated cytokine production. Lehner et al ${ }^{[25]}$ pointed out that enhanced host defense against infection was due to an improved peritoneal accumulation of neutrophilic granulocytes during the course of LPS pretreatment and an increase in Kupffer cell numbers and their phagocytic capacity in the liver. These results suggested that the "preactivated" immunity might be associated with the enhanced anti-bacterial function in endotoxin tolerant animals.

Recently, several papers were published on the impact of endotoxin tolerance on the functions of innate immune cells. Endotoxin tolerance significantly improved phagocytosis ${ }^{[26,27]}$ and impaired antigen presentation by macrophages ${ }^{[27]}$ in vitro. Wysoka et al ${ }^{[28]}$ demonstrated that DCs loss and inhibition of IL-12 production took place after a single high dose LPS stimulus. However, little is known about the functions and alterations of DCs during repetitive low-dose LPS pretreatment.

A number of previous studies have shown that DCs are the major source of early IL-12 production after in vivo exposure to LPS, which plays a central role in the pathogenesis of LPS-associated sepsis by stimulating IFN- $\gamma$ production. High levels of IL-12 in the microenvironment enhance the secretion of other proinflammatory cytokines and chemokines, as well as the recruitment and/or activation of other effectors, which ultimately leads to Th1 response and a potent inflammatory reaction ${ }^{[15,29,30]}$. Moreover, neutralization of IL-12 protects mice from lethal endotoxemia. The results in the present study are in accordance with the observations of previous in vitro and in vivo studies ${ }^{[31,32]}$, proving a remarkable inhibition of IL-12 production by DCs from endotoxin tolerant mice.

The increase in the uptake of dextran suggested that DCs from endotoxin tolerant mice had enhanced endocytotic ability. DCs take up self and nonself antigens by endocytosis and then generate and maintain specific immune responses by processing and presenting antigens to $T$ cells. Thus, the endocytosis of potentially dangerous microbes by DCs constitutes the first line of defense against pathogens. The enhanced endocytotic ability of DCs, together with other phagocytes, including macrophages ${ }^{[25-27]}$, during repetitive low-dose LPS pretreatment may help in the clearance of pathogens.

The DCs are among the most potent professional antigen-presenting cells that can initiate the adaptive immune response against microorganisms. To be able to initiate an immune response, DCs must express microbial peptides in the context of MHC-II molecules on the cell surface and provide secondary signals through co-stimulatory molecules. The up-regulated MHC-II and CD80 on DCs from endotoxin tolerant mice indicated that direct interaction with LPS or bystander effects during consecutive low-dose LPS pretreatment put DCs into a "pre-activated" state and made them ready to confront the insult of pathogens. These findings differ from the observation in macrophages, which showed a down-regulation in the expression of HLA-DA ${ }^{[27]}$. Further studies, such as $\mathrm{T}$ cell proliferation assay, are needed to clarify the antigen presentation in DCs from endotoxin tolerant mice. In addition, maturing DCs should be taken into consideration when exploring the antigen presenting capacity in endotoxin tolerance.

It is notable that DCs from endotoxin tolerant mice expressed high levels of MHC-II and CD80, in contrast to their low levels of CD40 and CD86. Banchereau et al ${ }^{[33,34]}$ pointed out that the interaction between CD86 and its corresponding ligand $\mathrm{CD} 28$ on $\mathrm{T}$ cells resulted in the upregulation of CD40 ligand on $\mathrm{T}$ cells. The $\mathrm{T}$ cells may then engage CD40 on DCs and trigger a burst of cytokine expression, including IL-12. Therefore, the blockage of CD86 and CD40 up-regulation that was observed in our experiment may contribute to attenuated cytokine production in endotoxin tolerance. The difference between the expression of MHC-II/CD80 and CD86/CD40 might provide DCs with an augmented antigen presenting ability without the excessive secretion of pro-inflammation cytokines and mediators.

It is believed that precursor DCs enter tissues as immature cells that exhibit a high endocytotic capacity. Immature DCs can then develop into mature DCs by a process that is driven by inflammatory stimuli or microenviromental factors such as bacterial products, LPS, and locally produced 
cytokines. During maturation and migration, they downregulate their endocytotic activity in accordance with the expression of MHC-II molecules, as well as co-stimulatory molecules ${ }^{[35]}$. Interestingly, the bone marrow DCs isolated from in vivo endotoxin tolerance displayed a novel phenotype of enhanced endocytosis and selective up-regulation of MHC-II and CD80. This could be explained by the specific "reprogramming" during the low-dose LPS treatment, which pre-activated the immune cells. One recent study ${ }^{[36]}$ indicated that the "reprogramming" was associated with different epigenetic modulations between inflammatory genes and anti-infection genes. Another possibility is the complexity of the bone marrow DCs during in vitro isolation and culturing. Further investigation of DCs from peripheral immune tissue such as the spleen and lymph node is necessary.

In general, these observations suggested that during repetitive low-dose LPS pretreatment, DCs carried out a series of alterations that resulted in enhanced endocytotic ability and selective up-regulation of MHC-II and CD80 expression. The mechanisms that underlie these alterations might differ from the regulation of cytokine production.

\section{Acknowledgements}

This work was supported by the Youth Research Foundation of Fudan University (Z-16/30) and the Shanghai Leading Academic Discipline Project (B115). We thank Dr Chun-ming LIANG for helpful technical support on cell culture technique and flow cytometric analysis.

\section{Author contribution}

Jing ZHANG, Jie-ming QU, and Li-xian HE designed research; Jing ZHANG performed research; Jing ZHANG analyzed data; Jing ZHANG and Jie-ming QU wrote the paper.

\section{References}

1 Cazzola M, Matera MG, Pezzuto G. Inflammation-a new therapeutic target in pneumonia. Respiration 2005; 72: 117-26.

2 Morrison DC, Ryan JL. Endotoxins and disease mechanisms. Annu Rev Med 1987; 38: 417-32.

3 Miyake K. Endotoxin recognition molecules MD-2 and tolllike receptor 4 as potential targets for therapeutic intervention of endotoxin shock. Curr Drug Targets Inflamm Allergy 2004; 3: 291-7.

4 Axtelle T, Pribble J. An overview of clinical studies in healthy subjects and patients with severe sepsis with IC14, a CD14-specific chimeric monoclonal antibody. J Endotoxin Res 2003; 9: 385-9.

5 West MA, Heagy W. Endotoxin tolerance: a review. Crit Care
Med 2002; 30: S64-73.

6 El Gazzar M, Yoza BK, Hu JY, Cousart SL, McCall CE. Epigenetic silencing of tumor necrosis factor alpha during endotoxin tolerance. J Biol Chem 2007; 282: 26857-64.

7 Chae BS. Comparative study of the endotoxemia and endotoxin tolerance on the production of Th cytokines and macrophage interleukin-6: differential regulation of indomethacin. Arch Pharm Res 2002; 25: 910-6.

$8 \mathrm{Qu} \mathrm{J}$, Zhang J, Pan J, He L, Ou Z, Zhang X, et al. Endotoxin tolerance inhibits lipopolysaccharide-initiated acute pulmonary inflammation and lung injury in rats by the mechanism of nuclear factor-kappaB. Scand J Immunol 2003; 58: 613-9.

9 Sanchez-Cantu L, Rode HN, Christou NV. Endotoxin tolerance is associated with reduced secretion of tumor necrosis factor. Arch Surg 1989;124:1432-5; discussion 1435-6.

10 Flohe S, Dominguez Fernandez E, Ackermann M, Hirsch T, Borgermann J, Schade FU. Endotoxin tolerance in rats: expression of TNF-alpha, IL-6, IL-10, VCAM-1 and HSP70 in lung and liver during endotoxin shock. Cytokine 1999; 11: 796-804.

11 Zingarelli B, Hake PW, Cook JA. Inducible nitric oxide synthase is not required in the development of endotoxin tolerance in mice. Shock 2002; 17: 478-84.

12 Lehner MD, Hartung T. Endotoxin tolerance-mechanisms and beneficial effects in bacterial infection. Rev Physiol Biochem Pharmacol 2002; 144: 95-141.

13 Rayhane N, Fitting C, Lortholary O, Dromer F, Cavaillon JM. Administration of endotoxin asociated with lipopolysaccharide tolerance protects mice against fungal infection. Infect Immun 2000; 68: 3748-53.

14 Ueno H, Klechevsky E, Morita R, Aspord C, Cao T, Matsui T, et al. Dendritic cell subsets in health and disease. Immunol Rev 2007; 219: 118-42.

15 Montoya M, Edwards MJ, Reid DM, Borrow P. Rapid activation of spleen dendritic cell subsets following lymphocytic choriomeningitis virus infection of mice: analysis of the involvement of type 1 IFN. J Immunol 2005; 174: 1851-61.

16 Bozza S, Gaziano R, Spreca A, Bacci A, Montagnoli C, di Francesco P, et al. Dendritic cells transport conidia and hyphae of Aspergillus fumigatus from the airways to the draining lymph nodes and initiate disparate Th responses to the fungus. J Immunol 2002; 168: 1362-71.

17 Pulendran B, Palucka K, Banchereau J. Sensing pathogens and tuning immune responses. Science 2001; 293: 253-6.

18 Pulendran B. Variegation of the immune response with dendritic cells and pathogen recognition receptors. J Immunol 2005; 174: 2457-65.

19 Inaba K, Inaba M, Romani N, Aya H, Deguchi M, Ikehara S, et al. Generation of large numbers of dendritic cells from mouse bone marrow cultures supplemented with granulocyte/macrophage colony-stimulating factor. J Exp Med 1992; 176: 1693-702.

20 Mathison JC, Virca GD, Wolfson E, Tobias PS, Glaser K, Ulevitch RJ. Adaptation to bacterial lipopolysaccharide controls lipopolysaccharide-induced tumor necrosis factor production in rabbit macrophages. J Clin Invest 1990; 85: 1108-18.

21 Wilhelm W, Grundmann U, Rensing H, Werth M, Langemeyer J, Stracke $\mathrm{C}$, et al. Monocyte deactivation in severe human sepsis or following cardiopulmonary bypass. Shock 2002; 17: 354-60.

22 Heagy W, Nieman K, Hansen C, Cohen M, Danielson D, West 
MA. Lower levels of whole blood LPS-stimulated cytokine release are associated with poorer clinical outcomes in surgical ICU patients. Surg Infect (Larchmt) 2003; 4: 171-80.

23 Cavaillon JM, Adib-Conquy M. Bench-to-bedside review: endotoxin tolerance as a model of leukocyte reprogramming in sepsis. Crit Care 2006; 10: 233-41.

24 Wang XD, Qu JM, Zhang J, Ou ZL, Pan J. Inhibition of Pseudomonas aeruginosa initiated lung inflammation in endotoxin tolerant rats. Fudan Acad J (Medical Version) 2005; 32: 13-6. Chinese.

25 Lehner MD, Ittner J, Bundschuh DS, van Rooijen N, Wendel A, Hartung T. Improved innate immunity of endotoxin-tolerant mice increases resistance to Salmonella enterica Serovar Typhimurium infection despite attenuated cytokine response. Infect Immun 2001; 69: 463-71.

26 Wheeler DS, Lahni PM, Denenberg AG, Poynter SE, Wong $\mathrm{HR}$, Cook JA, et al. Induction of endotoxin tolerance enhances bacterial clearance and survival in murine polymicrobial sepsis. Shock 2008; 30: 267-73.

27 Muthukuru M, Cutler CW. Antigen capture of Porphyromonas gingivalis by human macrophages is enhanced but killing and antigen presentation are reduced by endotoxin tolerance. Infect Immun 2008; 76: 477-85.

28 Wysocka M, Robertson S, Riemann H, Caamano J, Hunter C, Mackiewicz A, et al. IL-12 suppression during experimental endotoxin tolerance: dendritic cell loss and macrophage hyporesponsiveness. J Immunol 2001; 166: 7504-13.
29 Kang BY, Kim E, Kim TS. Regulatory mechanisms and their therapeutic implications of interleukin-12 production in immune cells. Cell Signal 2005; 17: 665-73.

30 Nakahara T, Moroi Y, Uchi H, Furue M. Differential role of MAPK signaling in human dendritic cell maturation and Th1/Th2 engagement. J Dermatol Sci 2006; 42: 1-11.

31 Merlin T, Sing A, Nielsen PJ, Galanos C, Freudenberg MA. Inherited IL-12 unresponsiveness contributes to the high LPS resistance of the Lps(d) C57BL/10ScCr mouse. J Immunol 2001; 166: 566-73.

32 Balkhy HH, Heinzel FP. Endotoxin fails to induce IFN-gamma in endotoxin-tolerant mice: deficiencies in both IL-12 heterodimer production and IL-12 responsiveness. J Immunol 1999; 162: 3633-8.

33 Banchereau J, Briere F, Caux C, Davoust J, Lebecque S, Liu YJ, et al. Immunobiology of dendritic cells. Annu Rev Immunol 2000; 18: 767-811.

34 Banchereau J, Steinman RM. Dendritic cells and the control of immunity. Nature 1998; 392: 245-52.

35 Alvarez D, Vollmann EH, von Andrian UH. Mechanisms and consequences of dendritic cells migration. Immunity 2008; 29: 325-42.

36 Foster SL, Hargreaves DC, Medzhitov HR. Gene-specific control of inflammation by TLR-induced chromatin modifications. Nature 2007; 447: 972-8. Corrected from: Nature 2008; 451: 102. 